\title{
ACAROLOGY
}

\section{Ocorrência e Flutuação Populacional de Ácaros Associados a Seringais Vizinhos de Fragmentos de Cerrado}

\author{
Peterson R. Demite ${ }^{1}$ e Reinaldo J.F. Feres ${ }^{2}$ \\ ${ }^{1}$ Programa de Pós-Graduação em Biologia Animal, UNESP, S. José do Rio Preto, SP, Bolsista \\ Plantações E. Michelin Ltda., peterson_demite@yahoo.com.br \\ ${ }^{2}$ Depto. Zoologia e Botânica, Univ. Estadual Paulista - UNEESP, 15054-000, S. José do Rio Preto, SP \\ reinaldo@ibilce.unesp.br
}

Neotropical Entomology 36(1):117-127 (2007)

Occurrence and Fluctuation of Mite Populations Associated with Rubber Tree Crop Neighbor of Fragments of Cerrado

\begin{abstract}
The basic knowledge of the seasonal occurrence of mites can supply data for elaboration of programs of ecological management to be implanted with success in the future. The objective of this study was to determine the species richness and the seasonality of mites present in two areas of rubber tree crops neighboring to native areas in Itiquira, MT. Along one year, 25 quantitative samplings were accomplished in rubber tree crop neighboring to two fragments of Cerrado (Cerradão and Mata Ripária). There were registered 199,380 mites, of 48 species, belonging to 15 families. Of those, 13 species are phytophagous, 18 predators and 17 mycophagous or of unknown alimentary habit. Three phytophagous species represented more than $97 \%$ of the mites collected: Phyllocoptruta seringueirae Feres (80.8\%), Tenuipalpus heveae Baker (12.7\%) and Calacarus heveae Feres (3.6\%). Among the predators, the most abundant species were Agistemus sp., Scirula sp. and Euseius concordis (Chant). Twenty-eight species were common to both crops. The families that had the largest number of species collected in the neighboring area to Cerradão were Tydeidae (7), Tarsonemidae (6), Eriophyidae and Phytoseiidae (4), and in the area close to Mata Ripária, Tydeidae (9) and Phytoseiidae (8). The presence of vegetation near the crop should explain the great number of species of mites classified as accidental found in this study. The largest abundances and species richness occurred in the end of the rainy season and beginning of the dry season.
\end{abstract}

KEY WORDS: Acari, mite fauna, agroecosystem, biodiversity, Hevea brasiliensis

RESUMO - O conhecimento básico da ocorrência sazonal dos ácaros pode fornecer dados para elaboração de programas de manejo ecológico a serem implantados com sucesso no futuro. O objetivo deste estudo foi determinar a riqueza de espécies e a sazonalidade da acarofauna presente em dois campos de seringueira vizinhos de áreas nativas, no município de Itiquira, MT. Ao longo de um ano, foram realizadas 25 coletas quantitativas em seringueiras vizinhas a dois fragmentos de Cerrado (Cerradão e Mata Ripária). Foram registrados 199.380 ácaros, de 48 espécies, pertencentes a 15 famílias. Dessas, 13 espécies são fitófagas, 18 predadoras e 17 micófagas ou de hábito alimentar desconhecido. Três espécies fitófagas representaram mais de $97 \%$ dos ácaros coletados: Phyllocoptruta seringueirae Feres (80,8\%), Tenuipalpus heveae Baker (12,7\%) e Calacarus heveae Feres (3,6\%). Entre os predadores, os mais abundantes foram Agistemus sp., Scirula sp. e Euseius concordis (Chant). Vinte e oito espécies foram comuns aos dois cultivos. As famílias que tiveram o maior número de espécies coletadas na área vizinha ao Cerradão foram, Tydeidae (7), Tarsonemidae (6), Eriophyidae e Phytoseiidae (4) e, na área próxima à Mata Ripária, Tydeidae (9) e Phytoseidae (8). A presença de vegetação vizinha aos seringais deve ser a causa do grande número de espécies de ácaros classificadas como acidentais encontradas neste estudo. As maiores abundâncias e riquezas de espécies ocorreram no final do período chuvoso e início da estação seca.

PALAVRAS-CHAVE: Acari, acarofauna, agroecossistema, biodiversidade, Hevea brasiliensis 
A seringueira [Hevea brasiliensis (Willd. ex Adr. De Juss.) Müell. Arg., Euphorbiaceae], é uma planta lactescente de 20-30 m de altura, nativa da Região Amazônica, ocorrendo nas margens de rios e lugares inundáveis da mata de terra firme (Lorenzi 2000). Seu cultivo é de grande importância econômica, sendo amplamente cultivada nos estados de São Paulo, Mato Grosso, Bahia e Espírito Santo (IAC 2004).

Vários trabalhos relacionam espécies de ácaros associados à seringueira. Estudos como os de Feres (2000), Ferla \& Moraes (2002) e Hernandes \& Feres (2006) apresentam importantes dados sobre a diversidade, período de ocorrência, picos de abundância populacional e micro-hábitats ocupados.

Ferla \& Moraes (2002) registraram 41 espécies de ácaros em seringueiras no Mato Grosso, sendo Calacarus heveae Feres (Actinedida: Eriophyidae) e Phyllocoptruta seringueirae Feres (Actinedida: Eriophyidae) as espécies mais abundantes e freqüentes.

C. heveae é o ácaro-praga mais importante da cultura da seringueira. Essa espécie atinge grandes populações nos monocultivos do noroeste paulista e do Mato Grosso, ao final da estação chuvosa e início da estação seca (Vieira \& Gomes 1999, Feres et al. 2002, Ferla \& Moraes 2002). Esse eriofiídeo causa o bronzeamento e queda prematura de folhas (Feres 1992) e provocou, nos meses de maio e junho de 1997, o desfolhamento prematuro de até $75 \%$ da copas em plantas do clone RRIM 600, em Reginópolis, SP (Vieira \& Gomes 1999). Segundo Feres (2000), alguns produtores estimam que C. heveae ocasina perdas de até $30 \%$ na produção de látex.

$\mathrm{O}$ conhecimento básico em relação à ocorrência sazonal dos ácaros pode fornecer dados para elaboração de programas de controle biológico a serem implantados com sucesso no futuro.

Este trabalho teve como objetivos determinar a riqueza de espécies e a sazonalidade da acarofauna presente em dois cultivos de seringueira vizinhos de fragmentos de Cerrado, no município de Itiquira, MT.

\section{Material e Métodos}

O estudo foi desenvolvido em duas áreas de cultivo de seringueira do clone PB 235, vizinhas de dois fragmentos de Cerrado (sensu Ribeiro \& Walter 1998): Cerradão (17 ${ }^{\circ} 23^{\prime} \mathrm{S}$, $\left.54^{\circ} 42^{\prime} \mathrm{W}\right)$ e Mata Ripária $\left(17^{\circ} 22^{\prime} \mathrm{S}, 54^{\circ} 41^{\prime} \mathrm{W}\right)$, distantes $1100 \mathrm{~m}$ uma da outra, no município de Itiquira, sul do Mato Grosso.

A região está localizada no domínio do Cerrado, cujo clima predominante é o tropical sazonal, classificado como Aw de Köppen, de inverno seco (Ribeiro \& Walter 1998). A temperatura média anual é de $22-23^{\circ} \mathrm{C}$, com máxima absoluta podendo alcançar os $40^{\circ} \mathrm{C}$. A precipitação média é de $1200 \mathrm{~mm}$ a $1800 \mathrm{~mm}$, com a média mensal apresentando uma grande estacionalidade, concentrando-se nos meses de outubro a abril (estação chuvosa). Nos meses de maio a setembro (estação seca), os índices pluviométricos mensais podem chegar a zero (Coutinho 2002, Ab'Saber 2003).

As áreas estudadas não receberam nenhum tipo de tratamento químico ou biológico durante o período de desenvolvimento do estudo.
Foram estabelecidos cinco transectos, distantes $50 \mathrm{~m}$ entre si, desde a borda no limite com as áreas nativas, até 200 $\mathrm{m}$ no interior do seringal. Em cada transecto foram marcadas cinco plantas distantes cerca de $25 \mathrm{~m}$ entre si, totalizando 25 plantas por área de estudo. Em cada uma das 25 coletas realizadas com intervalo médio de 14 dias, de março de 2004 a março de 2005, foram coletadas sete folhas de cada planta ao redor da copa, até a altura de sete a oito metros.

Para o transporte aéreo até São José do Rio Preto, SP, as folhas foram acondicionadas em sacos de papel, no interior de sacos de polietileno, colocados em caixas isotérmicas de poliestireno com Gelo- $\mathrm{X}^{\circledR}$ em seu interior. No laboratório, o material foi armazenado sob refrigeração a $10^{\circ} \mathrm{C}$, por período máximo de sete dias. Foi analisado um folíolo de cada folha (totalizando 175 folíolos/área) sendo os ácaros montados em lâminas de microscopia, sob microscópio estereoscópico, utilizando-se o meio de Hoyer. Os ácaros oribatídeos foram triados e armazenados em álcool etílico a 70\%.

Nos períodos de grandes índices populacionais, os espécimes de $C$. heveae, $P$. seringueirae e Tenuipalpus heveae Baker (Actinedida: Tenuipalpidae) foram contados diretamente nos folíolos, devido ao seu fácil reconhecimento. Uma parcela dos ácaros de cada espécie (50 indivíduos por transecto/coleta) sempre foi montada para a confirmação da identificação.

A constância foi calculada segundo Bodenheimer (1955) apud Silveira-Neto et al. (1976) e as espécies classificadas, de acordo com sua freqüência nas amostras, em constantes $(\mathrm{C}>$ $50 \%)$, acessórias $(25<\mathrm{C}<50 \%)$ e acidentais $(\mathrm{C}<25 \%)$.

Os dados climáticos (temperatura, umidade relativa do ar e horas de sol) foram obtidos através da estação metereológica da empresa "Plantações E. Michelin Ltda.", localizada a 3860 $\mathrm{m}$ da área próxima ao fragmento de Cerradão e a $4760 \mathrm{~m}$ da área próxima ao fragmento de Mata Ripária. A pluviosidade foi obtida de um posto de coleta localizado mais próximo das duas áreas: $2820 \mathrm{~m}$ do fragmento de Cerradão e $3780 \mathrm{~m}$ da Mata Ripária.

O coeficiente de correlação de Spearman (rs), utilizado para verificar a possível relação dos ácaros com os dados climáticos registrados nos locais de estudo, foi calculado com o software BioEstat 3.0 ${ }^{\circledR}$ (Ayres et al. 2003).

O material testemunho foi depositado na coleção de Acari (DZSJRP)- http://www.splink.cria.org.br, do Departamento de Zoologia e Botânica, Universidade Estadual Paulista (UNESP), São José do Rio Preto, SP.

\section{Resultados}

No total, foram registrados 199.380 ácaros, de 48 espécies pertencentes a 15 famílias (Tabela 1). Dessas, 13 espécies são fitófagas, 18 predadoras e 17 micófagas ou de hábito alimentar desconhecido. Três espécies fitófagas representaram mais de $97 \%$ dos ácaros coletados: $P$. seringueirae (161.228 indivíduos - 80,8\%), T. heveae (25.390 indivíduos - 12,7\%) e C. heveae, (7.104 indivíduos - 3,6\%). Os fitófagos representaram 98,7\% do total de indivíduos coletados, enquanto que os predadores $1 \%$. Outros fitófagos abundantes foram Shevtchenkella petiolula Feres (Actinedida: Eriophyidae) (1.754 indivíduos) e Oligonychus 
Tabela 1. Abundância e constância das espécies de ácaros registradas em seringais vizinhos aos fragmentos de Cerrado (Cerradão e Mata Ripária), em Itiquira, MT.

\begin{tabular}{|c|c|c|c|c|c|c|}
\hline \multirow{3}{*}{ Espécies } & \multirow{3}{*}{ H.A. } & \multicolumn{4}{|c|}{ Seringueiras } & \multirow{3}{*}{$\begin{array}{c}\text { Total } \\
\mathrm{Ab} .\end{array}$} \\
\hline & & \multicolumn{2}{|c|}{ Cerradão } & \multicolumn{2}{|c|}{ Mata Ripária } & \\
\hline & & $\mathrm{Ab}$. & $\mathrm{C}$ & $\mathrm{Ab}$. & $\mathrm{C}$ & \\
\hline \multicolumn{7}{|l|}{ Acaridae } \\
\hline Caloglyphus sp. & M & 2 & Acid. & 164 & Acess. & 166 \\
\hline \multicolumn{7}{|l|}{ Ascidae } \\
\hline Asca sp. & $\mathrm{P}$ & - & - & 2 & Acid. & 2 \\
\hline \multicolumn{7}{|l|}{ Bdellidae } \\
\hline Hexabdella cf. singula & $\mathrm{P}$ & 1 & Acid. & 1 & Acid. & 2 \\
\hline \multicolumn{7}{|l|}{ Cheyletidae } \\
\hline Cheletomimus duosetosus Muma & $\mathrm{P}$ & - & - & 173 & Const. & 173 \\
\hline Cheletomimus aff. darwinia & $\mathrm{P}$ & - & - & 1 & Acid. & 1 \\
\hline \multicolumn{7}{|l|}{ Cunaxidae } \\
\hline Scirula sp. & $\mathrm{P}$ & 9 & Acess. & 309 & Const. & 318 \\
\hline Neocunaxoides sp. & $\mathrm{P}$ & 1 & Acid. & - & & 1 \\
\hline \multicolumn{7}{|l|}{ Eriophyidae } \\
\hline Cf. Acarolox sp. & $\mathrm{F}$ & 1 & Acid. & - & & 1 \\
\hline Calacarus heveae Feres & $\mathrm{F}$ & 1.076 & Const. & 6.028 & Const. & 7.104 \\
\hline Phyllocoptruta seringueirae Feres & $\mathrm{F}$ & 60.412 & Const. & 100.816 & Const. & 161.228 \\
\hline Shevtchenkella petiolula Feres & $\mathrm{F}$ & 439 & Acid. & 1.315 & Acess. & 1.754 \\
\hline \multicolumn{7}{|l|}{ Oripodidae } \\
\hline Pirnodus sp. & M & 8 & Acid. & 1 & Acid. & 9 \\
\hline \multicolumn{7}{|l|}{ Phytoseiidae } \\
\hline Amblyseius acalyphus Denmark \& Muma & $\mathrm{PP}$ & - & - & 72 & Const. & 72 \\
\hline $\begin{array}{l}\text { Amblyseius neochiapensis Lofego, Moraes } \\
\text { \& McMurtry }\end{array}$ & PP & 5 & Acid. & 24 & Acess. & 29 \\
\hline Euseius citrifolius Denmark \& Muma & $\mathrm{PP}$ & 20 & Acid. & 15 & Acess. & 35 \\
\hline Euseius concordis (Chant) & $\mathrm{PP}$ & 215 & Const. & 59 & Acess. & 274 \\
\hline Galendromus sp. & PP & - & - & 1 & Acid. & 1 \\
\hline Iphiseiodes zuluagai Denmark \& Muma & PP & 50 & Acess. & 3 & Acid. & 53 \\
\hline Proprioseiopsis aff. jasmine & $\mathrm{PP}$ & - & - & 1 & Acid. & 1 \\
\hline Typhlodromips aff. amilus & PP & - & - & 129 & Const. & 129 \\
\hline Imaturos & & 103 & & 80 & & 183 \\
\hline \multicolumn{7}{|l|}{ Scheloribatidae } \\
\hline Scheloribates sp. & M & 1 & Acid. & 1 & Acid. & 2 \\
\hline \multicolumn{7}{|l|}{ Stigmaeidae } \\
\hline Agistemus sp. & $\mathrm{P}$ & 275 & Const. & 142 & Acess. & 417 \\
\hline Zetzellia quasagistemas Hernandes \& Feres & $\mathrm{P}$ & 38 & Acid. & 92 & Acess. & 130 \\
\hline Imaturos & & 66 & & 56 & & 122 \\
\hline \multicolumn{7}{|l|}{ Tarsonemidae } \\
\hline Daidalotarsonemus tesselatus DeLeon & $?$ & 150 & Acess. & 141 & Acess. & 291 \\
\hline
\end{tabular}


Tabela 1. Continuação.

\begin{tabular}{|c|c|c|c|c|c|c|}
\hline \multirow{3}{*}{ Espécies } & \multirow{3}{*}{ H.A. } & \multicolumn{4}{|c|}{ Seringueiras } & \multirow{3}{*}{$\begin{array}{c}\text { Total } \\
\mathrm{Ab} .\end{array}$} \\
\hline & & \multicolumn{2}{|c|}{ Cerradão } & \multicolumn{2}{|c|}{ Mata Ripária } & \\
\hline & & $\mathrm{Ab}$. & $\mathrm{C}$ & $\mathrm{Ab}$. & $\mathrm{C}$ & \\
\hline Deleonia sp. & $?$ & - & - & 1 & Acess. & 1 \\
\hline Tarsonemus confusus Ewing & M1 & 24 & Acess. & - & - & 24 \\
\hline Tarsonemus sp.1 & M1 & 22 & Acess. & 11 & Acid. & 33 \\
\hline Tarsonemus sp. 2 & $\mathrm{M}^{1}$ & 18 & Acess. & - & - & 18 \\
\hline Tarsonemus sp. 3 & $M^{1}$ & 1 & Acess. & - & - & 1 \\
\hline Xenotarsonemus sp. & $?$ & 1 & Acid. & 1 & Acid. & 2 \\
\hline Imaturos & & 64 & & 42 & & 106 \\
\hline \multicolumn{7}{|l|}{ Tenuipalpidae } \\
\hline Brevipalpus phoenicis (Geijskes) & $\mathrm{F}$ & 3 & Acid. & - & - & 3 \\
\hline Tenuipalpus heveae Baker & $\mathrm{F}$ & 10.642 & Const. & 14.748 & Const. & 25.390 \\
\hline \multicolumn{7}{|l|}{ Tetranychidae } \\
\hline Aponychus cf. shultzi & $\mathrm{F}$ & - & - & 1 & Acid. & 1 \\
\hline Eutetranychus banksi (McGregor) & $\mathrm{F}$ & 1 & Acid. & - & & 1 \\
\hline Oligonychus gossypii (Zacher) & $\mathrm{F}$ & 193 & Const. & 394 & Const. & 587 \\
\hline Tetranychus mexicanus (McGregor) & $\mathrm{F}$ & 64 & Const. & 214 & Const. & 278 \\
\hline Imaturos & & 47 & & 131 & & 178 \\
\hline \multicolumn{7}{|l|}{ Tydeidae } \\
\hline Lorryia formosa Cooreman & $\mathrm{F}^{2}$ & 6 & Acid. & 14 & Acess. & 20 \\
\hline Lorryia sp.1 & $\mathrm{F}$ & - & - & 2 & Acid. & 2 \\
\hline Lorryia sp2. & $\mathrm{F}$ & 14 & Acess. & 18 & Acid. & 32 \\
\hline Metapronematus sp. & $?$ & 1 & Acid. & 10 & Acid. & 11 \\
\hline Neolorryia boycei (Baker) & $?$ & 3 & Acid. & 6 & Acid. & 9 \\
\hline Paralorryia sp. & $?$ & 1 & Acid. & - & & 1 \\
\hline Parapronematus sp. & $\mathrm{P}^{3}$ & 1 & Acid. & 2 & Acid. & 3 \\
\hline Pretydeus sp. & $?$ & - & - & 1 & Acid. & 1 \\
\hline Pronematus sp. & $\mathrm{P}^{4}$ & - & - & 9 & Acid. & 9 \\
\hline Pseudolorryia sp. & $?$ & 80 & Acess. & 37 & Acess. & 117 \\
\hline Imaturos & & 8 & & 23 & & 31 \\
\hline \multicolumn{7}{|l|}{ Winterschmidtiidae } \\
\hline Czenspinskia sp. & $\mathrm{M}^{5}$ & 4 & Acid. & 1 & Acid. & 5 \\
\hline Oulenzia sp. & $\mathrm{M}^{5}$ & 3 & Acid. & 15 & Acid. & 18 \\
\hline Abundância & & 74.072 & & 125.308 & & 199.380 \\
\hline Riqueza & & 36 & & 40 & & 48 \\
\hline
\end{tabular}

(Ab) Abundância; (C) Constância: Const.: constantes; Acess.: acessórias; Acid.: acidentais; (H.A.) Hábito alimentar: (F) fitófagos (Jeppson et al. 1975); (PP) preponderantemente predadores (Krantz 1978, McMurtry \& Croft 1997); (P) predadores (Krantz 1978, Flechtmann 1986), (M) micófagos; (?) existem dúvidas a respeito do hábito alimentar dessas espécies ou gêneros: (1) Lindquist 1986; (2) Smirnoff 1957, Gerson 1968, Flechtmann 1973, Flechtmann \& Arleu 1984; (3) McCoy et al. 1967; (4) Baker 1965, Baker \& Wharton 1952, Bayan 1986, Calvert \& Huffaker 1974, Knop \& Hoy 1983, Laing \& Knop 1983, Perrin \& McMurtry 1996; (5) Baker \& Wharton 1952, Krantz 1978, Woolley 1988. 
gossypii (Zacher) (Actinedida: Tetranychidae) (587 indivíduos). Dentre os predadores, os mais abundantes foram Agistemus sp. (Actinedida: Stigmaeidae) (417 indivíduos), Scirula sp. (Actinedida: Cunaxidae) (318 indivíduos), Euseius concordis (Chant) (Gamasida: Phytoseiidae) (274 indivíduos) e Cheletomimus duosetosus Muma (Actinedida: Cheyletidae) (173 indivíduos).

Nas seringueiras vizinhas ao fragmento de Cerradão, foram coletados 74.072 ácaros de 36 espécies, pertencentes a 13 famílias. Nessa área, foram mais abundantes os fitófagos $P$. seringueirae, T. heveae, C. heveae, S. petiolula e O. gossypii e os predadores Agistemus sp. e E. concordis. Nas seringueiras próximas à Mata Ripária, foram coletados 125.308 ácaros, de 40 espécies pertencentes a 15 famílias, sendo as espécies fitófagas mais abundantes as mesmas do cultivo próximo ao fragmento de Cerradão, e os predadores mais abundantes Scirula sp. e C. duosetosus.

Vinte e oito espécies foram comuns às duas áreas estudadas. Oito espécies foram coletadas somente na área próxima ao fragmento de Cerradão, e 12 na área vizinha da Mata Ripária. A maioria das espécies coletadas em somente uma das áreas foi pouco abundante, com exceção dos predadores Amblyseius acalyphus Denmark \& Muma (Gamasida: Phytoseiidae), Typhlodromips aff. amilus (Gamasida: Phytoseiidae) e C. duosetosus que ocorreram nas seringueiras vizinhas à Mata Ripária.

A maioria das espécies coletadas nas duas áreas foi acidental: 55,6\% nas seringueiras próximas ao fragmento de Cerradão e 50\% nas vizinhas ao fragmento de Mata Ripária.

As famílias que tiveram o maior número de espécies coletadas na área vizinha ao fragmento de Cerradão foram Tydeidae (7 espécies), Tarsonemidae (6), Eriophyidae e Phytoseiidae (4). Nenhuma espécie de Tydeidae e Tarsonemidae foi classificada como constante. O fitoseídeo E. concordis foi classificado como constante e Iphiseiodes zuluagai Denmark \& Muma (Gamasida: Phytoseiidae) como acessória. Euseius citrifolius Denmark \& Muma (Gamasida: Phytoseiidae) e Amblyseius neochiapensis Lofego, Moraes \& McMurtry (Gamasida: Phytoseiidae) foram classificadas como acidentais. Dentre os eriofiídeos, $P$. seringueirae e $C$. heveae foram constantes, e $S$. petiolula acessória. As outras espécies classificadas como constantes nas seringueiras próximas ao fragmento de Cerradão foram $T$. heveae, $O$. gossypii e Tetranychus mexicanus (McGregor) (Actinedida: Tetranychidae).

$\mathrm{Na}$ área próxima ao fragmento de Mata Ripária, a família com maior riqueza também foi Tydeidae (9 espécies), seguida por Phytoseidae (8). Das espécies de tideídeos registradas nessa área, assim como no cultivo próximo ao fragmento de Cerradão, nenhuma foi classificada como constante, sendo Lorryia formosa Cooreman (Actinedida: Tydeidae) e Pseudolorryia sp. (Actinedida: Tydeidae) classificadas como acessórias. Dos fitoseídeos aí registrados, duas espécies foram classificadas como constantes (A. acalyphus e $T$. aff. amilus), três como acessórias (A. neochiapensis, E. citrifolius e E. concordis) e três acidentais [Galendromus sp., I. zuluagai e Proprioseiopsis cf. jasmini (Gamasida: Phytoseidae)]. Os fitófagos $P$. seringueirae, $C$. heveae, $T$. heveae, $O$. gossypii e T. mexicanus, e o predador Scirula sp. foram constantes.

As maiores abundâncias e riquezas ocorreram no final da estação chuvosa e as menores logo após o período de senescência (Figs. 1 e 2).

De março a junho a temperatura média foi de $23,5^{\circ} \mathrm{C}$ e a soma das chuvas alcançou $571,7 \mathrm{~mm}$, sendo mais concentradas nos meses de março e abril. Nas coletas realizadas nesses meses foi registrada riqueza média de 23 espécies, e mais de $75 \%$ dos ácaros foram registrados nesse período. Nos últimos meses (maio e junho), ocorreu diminuição na precipitação e temperatura (Fig. 3). Junho foi o mês em que se registrou a menor umidade relativa do ar.

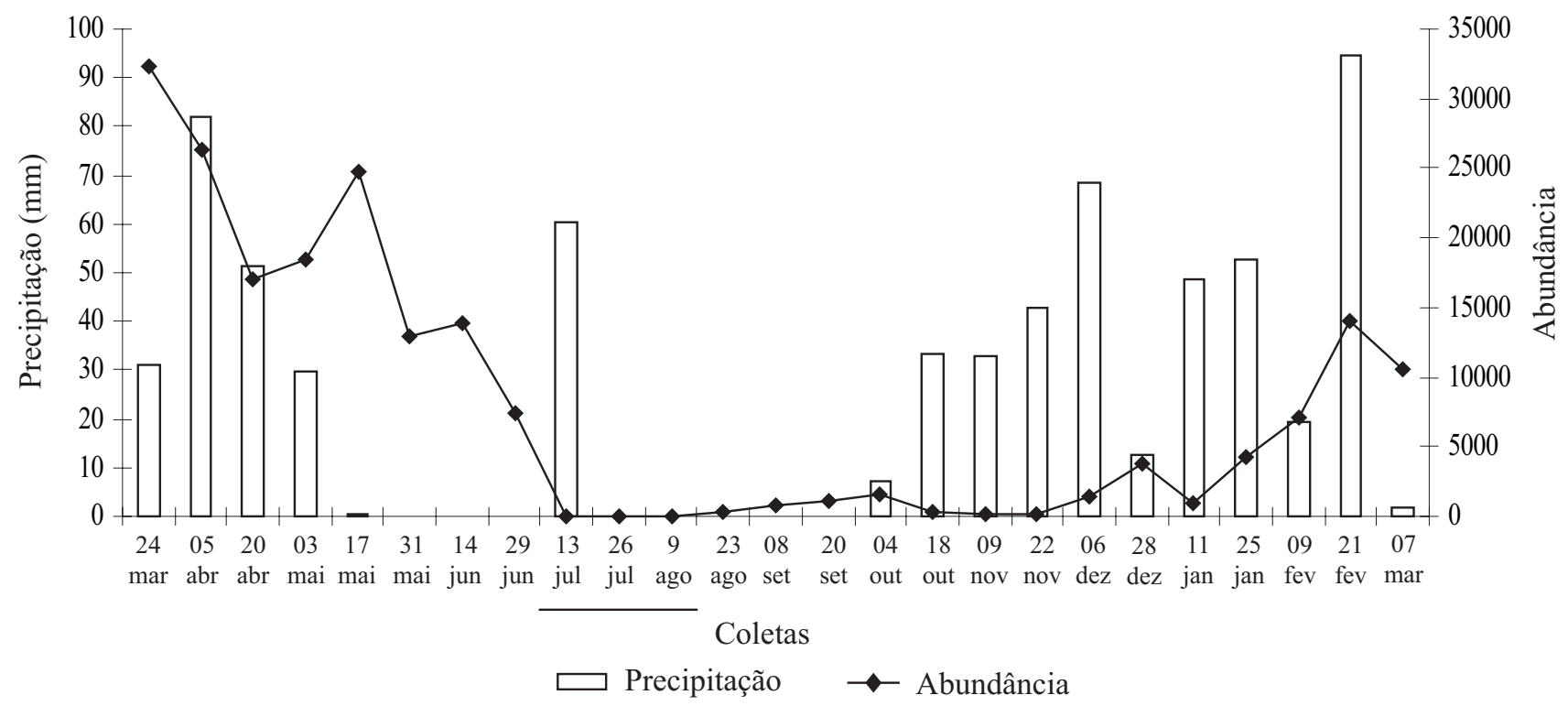

Fig. 1. Abundância de ácaros e pluviosidade registradas de março de 2004 a março de 2005 em Itiquira, MT. Barra: período de senescência. 


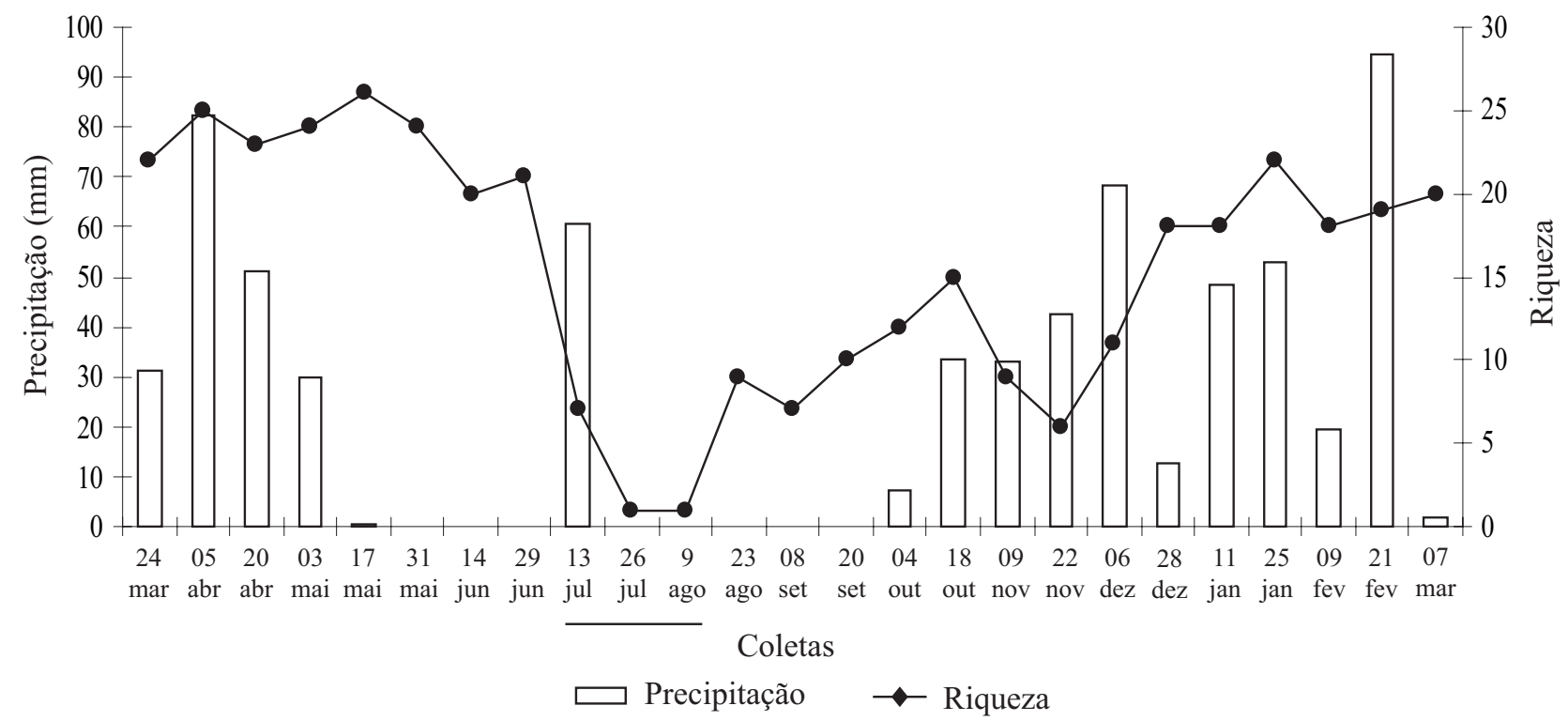

Fig. 2. Riqueza e pluviosidade registradas de março de 2004 a março de 2005 em Itiquira, MT. Barra: período de senescência.

A precipitação acumulada nos meses seguintes (julho, agosto, setembro e outubro), atingiu somente $234 \mathrm{~mm}$, ocorrendo estiagem de 65 dias entre julho a setembro. Em agosto registrou-se o maior número de horas de sol ao dia e a maior parte das chuvas registradas nesse período ocorreu no mês de outubro, com temperatura média de $23,2^{\circ} \mathrm{C}$. Em julho e início do mês de agosto ocorreu a queda natural das folhas, não sendo possível a amostragem em todos os transectos, e no final de julho não foi possível a coleta de nenhuma amostra. Após esse período a riqueza e a abundância foram baixas, só aumentando no final do mês de dezembro. Entre dezembro de 2004 a março de 2005 a soma das chuvas chegou a $1.134,8$ $\mathrm{mm}$. Nesse período a média da umidade relativa do ar ficou acima dos $81 \%$ e a temperatura média foi de $25,4^{\circ} \mathrm{C}$, com

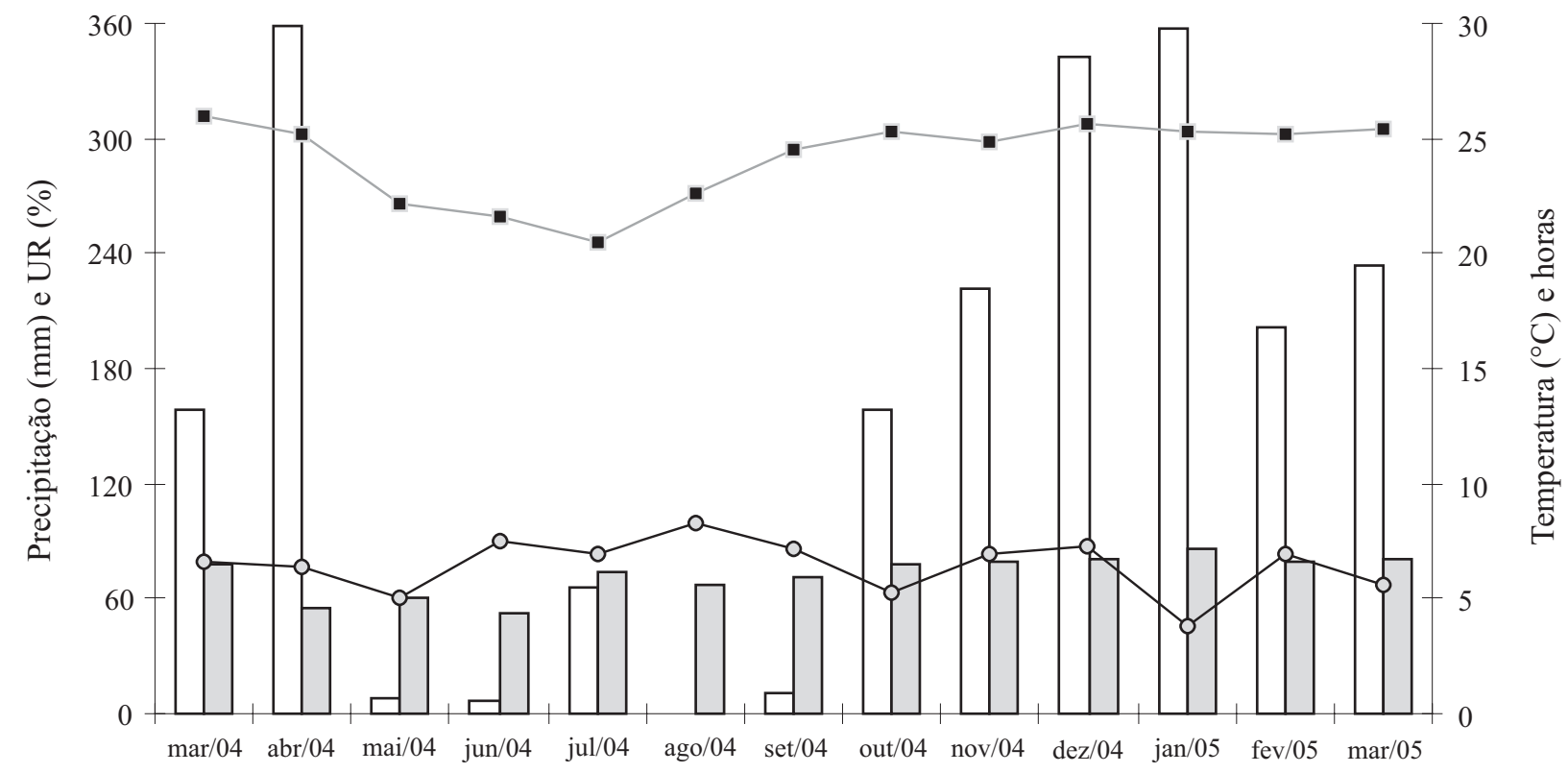

$\square$ Precipitação $\square$ UR média $\quad$ - Temperatura (média) —— Horas de sol

Fig. 3. Dados climáticos registrados de março de 2004 a março de 2005 em Itiquira, MT. 
riqueza média registrada de 18 espécies.

Verificou-se correlação significativa entre pluviosidade e as populações de $T$. heveae $(\mathrm{rs}=0,44 ; \mathrm{P}=0,04)$ e $O$. gossypii ( $\mathrm{rs}=0,60 ; \mathrm{P}=0,002)$, e marginalmente significativa com a de $C$. heveae ( $\mathrm{rs}=0,40 ; \mathrm{P}=0,06)$. Com relação à umidade, somente para $O$. gossypii $(\mathrm{rs}=0,46 ; \mathrm{P}=0,03)$ verificou-se correlação significativa. Para T. heveae a correlação com a temperatura foi significativa $(r s=0,46 ; P=0,03)$. Nenhuma das cinco espécies de fitófagos mais abundantes foram correlacionadas com as horas de sol ao dia.

Nas poucas amostras coletadas no mês de julho (período de senescência), e de outubro até dezembro, verificou-se um grande número de indivíduos e fezes de Leptopharsa heveae Drake \& Poor (Hemiptera: Tingidae) nas folhas das seringueiras próximas ao fragmento de Cerradão.

$P$. seringueirae foi mais abundante no início da estação seca (Fig. 4a) e T. heveae nos meses com as maiores precipitações registradas (Fig. 4b). S. petiolula e C. heveae, foram mais abundantes em setembro de 2004 e fevereiro de 2005 , respectivamente, e $O$. gossypii e T. mexicanus no início de dezembro e final de novembro, respectivamente (Fig. 4c).

Em relação aos predadores, Agistemus sp. e I. zuluagai foram mais abundantes no mês de junho (Fig. 5a), e $T$. aff. amilus, Zetzellia quasagistemas Hernandes e Feres (Actinedida: Stigmaeidae) e C. duosetosus em abril (Fig. 5b). E. concordis e A. acalyphus tiveram seus maiores níveis populacionais registrados nos meses de maio e dezembro, respectivamente. Scirula sp. foi mais abundante no final de março de 2004 e início de março de 2005 (Fig. 5c).

E. concordis e I. zuluagai juntamente com Agistemus sp., tiveram seus picos populacionais logo após os de $P$. seringueirae. Já $C$. duosetosus, T. aff. amilus e $Z$. quasagistemas ocorreram em maiores níveis populacionais durante o períodos de grande abundância do fitófago $P$. seringueirae. Estas espécies de predadores foram mais comuns ou exclusivas no seringal próximo à Mata Ripária. S. petiolula foi mais abundantes logo após o período de senescência. A maior abundância de Scirula sp. ocorreu no mesmo período de $C$. heveae e T. heveae.

\section{Discussão}

C. heveae é relatada como a espécie de ácaro mais abundante da seringueira no estado de São Paulo (Demite \& Feres 2005, Hernandes \& Feres 2006, Vis et al. 2006). Neste estudo, entretanto, outro eriofí́deo, P. seringueirae, e o tenuipalpídeo $T$. heveae foram os ácaros fitófagos que apresentaram a maior abundância nas duas áreas estudadas. Ferla \& Moraes (2002) já haviam registrado P. seringueirae como a espécie mais freqüente em seringais no município de Pontes e Lacerda, MT.

Agistemus sp. foi o ácaro predador mais abundante, diferentemente do que foi constatado no estado de São Paulo, onde os principais foram E. citrifolius e Z. quasagistemas (Bellini et al. 2005- relatado como Z. aff. yusti; Demite \& Feres 2005, Hernandes \& Feres 2006). Ácaros das famílias Cunaxidae e Cheyletidae são raramente encontrados na cultura da seringueira, entretanto, ácaros dessas duas famílias foram abundantes, principalmente nas seringueiras próximas ao fragmento de Mata Ripária. A diferença na composição da acarofauna (principalmente de predadores) em seringais do Mato Grosso em relação aos de São Paulo, pode estar relacionada com a vegetação da região. A presença de vegetação vizinha também deve explicar o grande número de espécies de ácaros classificadas neste trabalho como acidentais.

A maior abundância e riqueza registrada no final da estação chuvosa foram também observadas por Fazolin \& Pereira (1989) em seringais da Região Norte do Brasil e por Feres et al. (2002), Bellini et al. (2005), Hernandes \& Feres (2006) e Vis et al. (2006) no estado de São Paulo. Segundo Feres et al. (2002), a possível explicação é que a redução na precipitação favorece os ácaros, principalmente os que habitam a parte superior dos folíolos, já que eles sofrem menos o efeito mecânico das chuvas, que pode removê-los dos folíolos. Nesse período também ocorre maior disponibilidade de folhas em condições fisiológicas aparentemente favoráveis para os ácaros (Bellini et al. 2005). Entretanto, parece que a ocorrência de chuvas ocasionais favorece as populações de ácaros, mantendo a umidade relativa do ar elevada. Segundo Ferla \& Moraes (2003a), maiores níveis de umidade favorecem as populações de $C$. heveae.

$S$. petiolula foi a espécie mais abundante logo após a senescência, sugerindo que as populações desta espécie se desenvolvem melhor nos pecíolos de folhas jovens, como também no período mais seco do ano. Feres (2001) e Vis et al. (2006), também verificaram a ocorrência dessa espécie no estado de São Paulo nesse mesmo período.

O pequeno número de ácaros nas primeiras coletas após a senescência se deve ao pouco tempo para recolonização. Segundo Hernandes \& Feres (2006), além desse fato, outro motivo que pode influenciar o pequeno número de ácaros nesse período é a presença de folhas jovens. Essas folhas possuem diferenças nas concentrações de componentes químicos quando comparadas com folhas velhas (Wendler et al. 1995, Valjakka et al. 1999).

O pequeno número de fitófagos amostrado em meados de julho pode estar relacionado ao processo de senescência. A condição fisiológica das folhas deve ter interferido na sua ocorrência. Nesse período, não foi possível a amostragem das folhas de todos os transectos. Nas poucas amostras obtidas, ocorreram indivíduos de $L$. heveae, uma das principais pragas da cultura da seringueira (Pereira \& Carvalho Pereira 2001). A presença de $L$. heveae no período de outubro a dezembro pode ter interferido na riqueza e abundância de ácaros fitófagos nas seringueiras próximas ao fragmento de Cerradão, provocando sua redução. Folhas com indivíduos adultos, imaturos e fezes desse inseto apresentaram várias machas claras, sintoma de ataque desse inseto. Tanto o adulto quanto a ninfa, sugam a seiva das plantas, diminuindo sua atividade fotossintética e, conseqüentemente, debilitando-as (Vendramim 1992).

Os maiores níveis populacionais de C. duosetosus, $T$. aff. amilus e $Z$. quasagistemas coincidindo com os de $P$. seringueirae e $T$. heveae, indicam que esses predadores podem se alimentar desses fitófagos. Hernandes \& Feres (2006) verificaram a presença de Z. quasagistemas no período de maior abundância de $C$. heveae e $T$. heveae. Bellini et al. (2005) sugeriram que esse predador pode contribuir para 


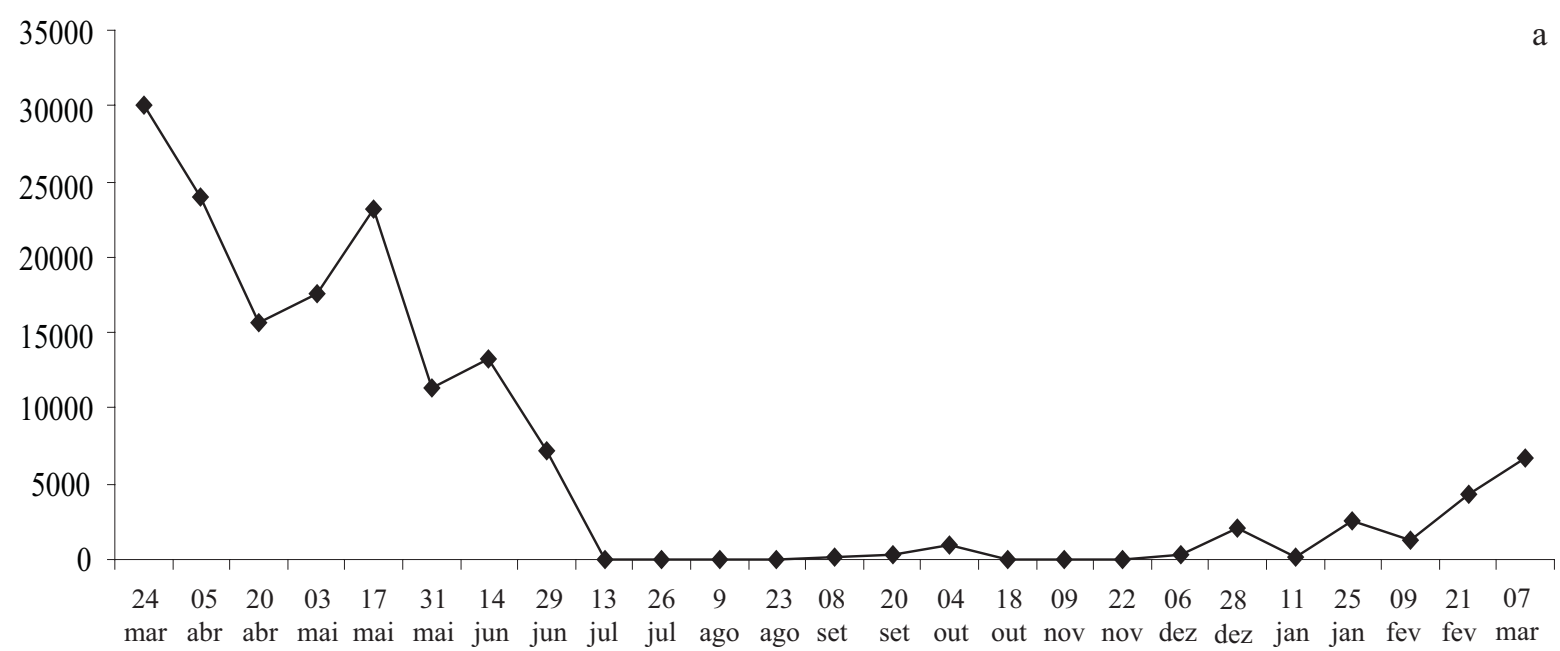

$\leftrightarrow$ Phyllocoptruta seringueirae
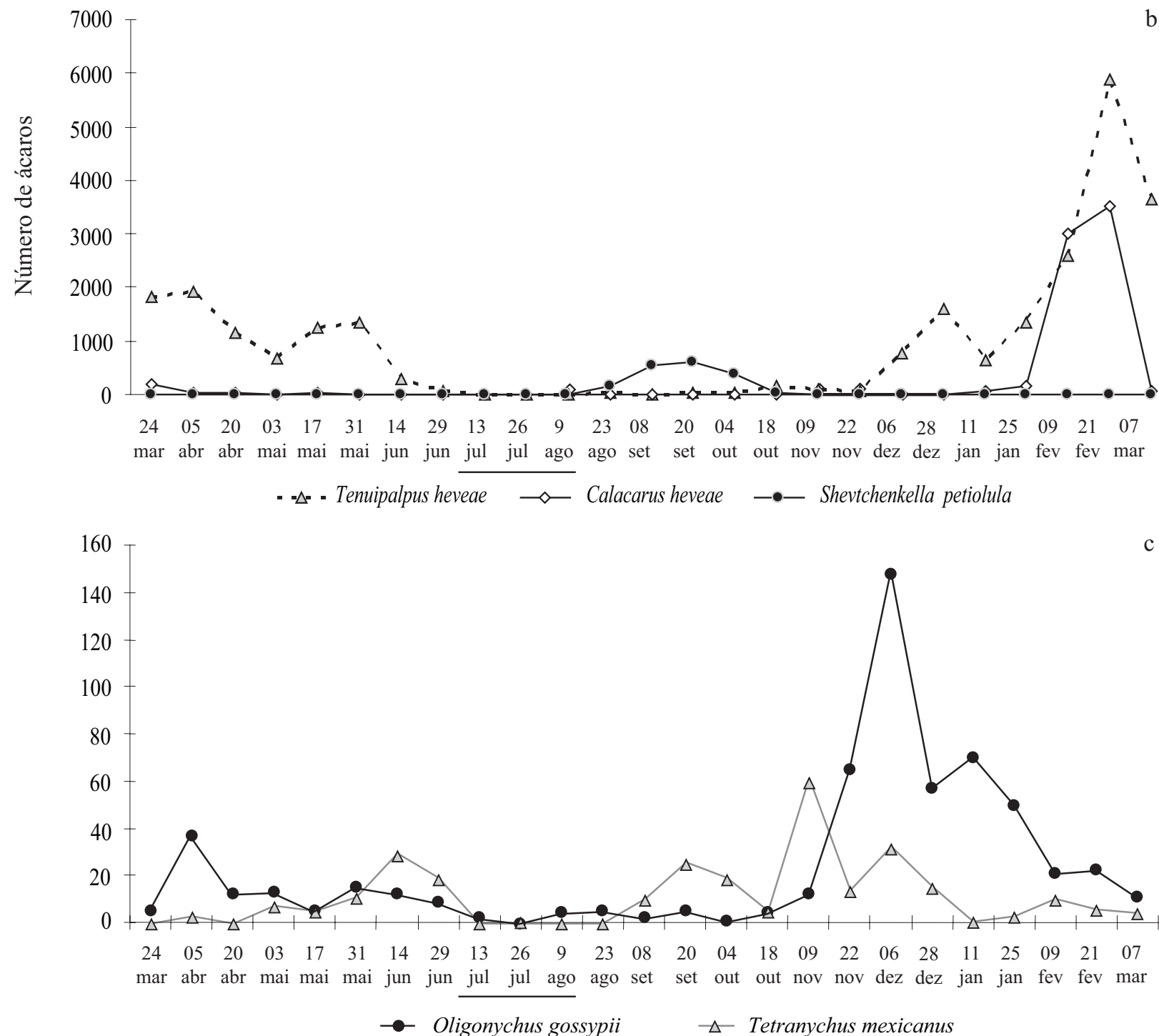

Fig. 4. Flutuação populacional dos fitófagos mais abundantes nos dois cultivos de seringueira de março de 2004 a março de 2005 em Itiquira, MT. Barra: período de senescência. 


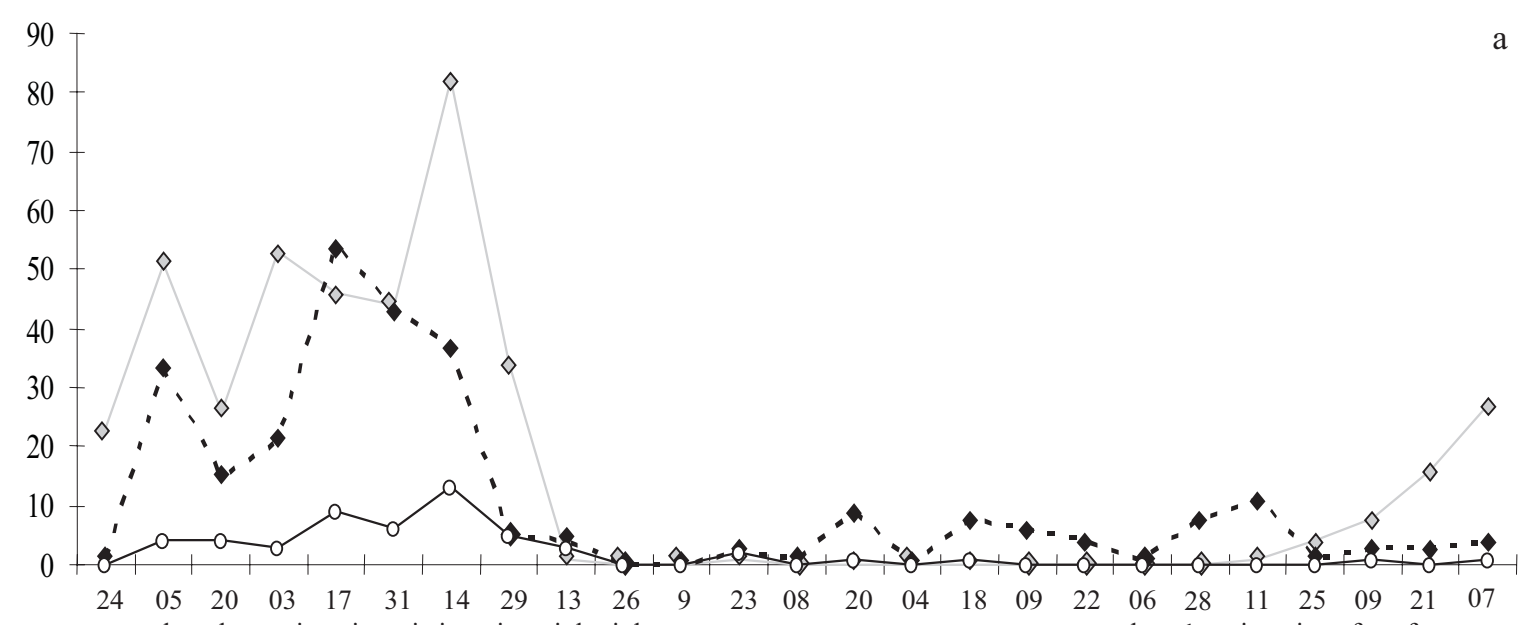
mar abr abr mai mai mai jun jun jul jul ago ago set set out out nov nov dez dez jan jan fev fev mar

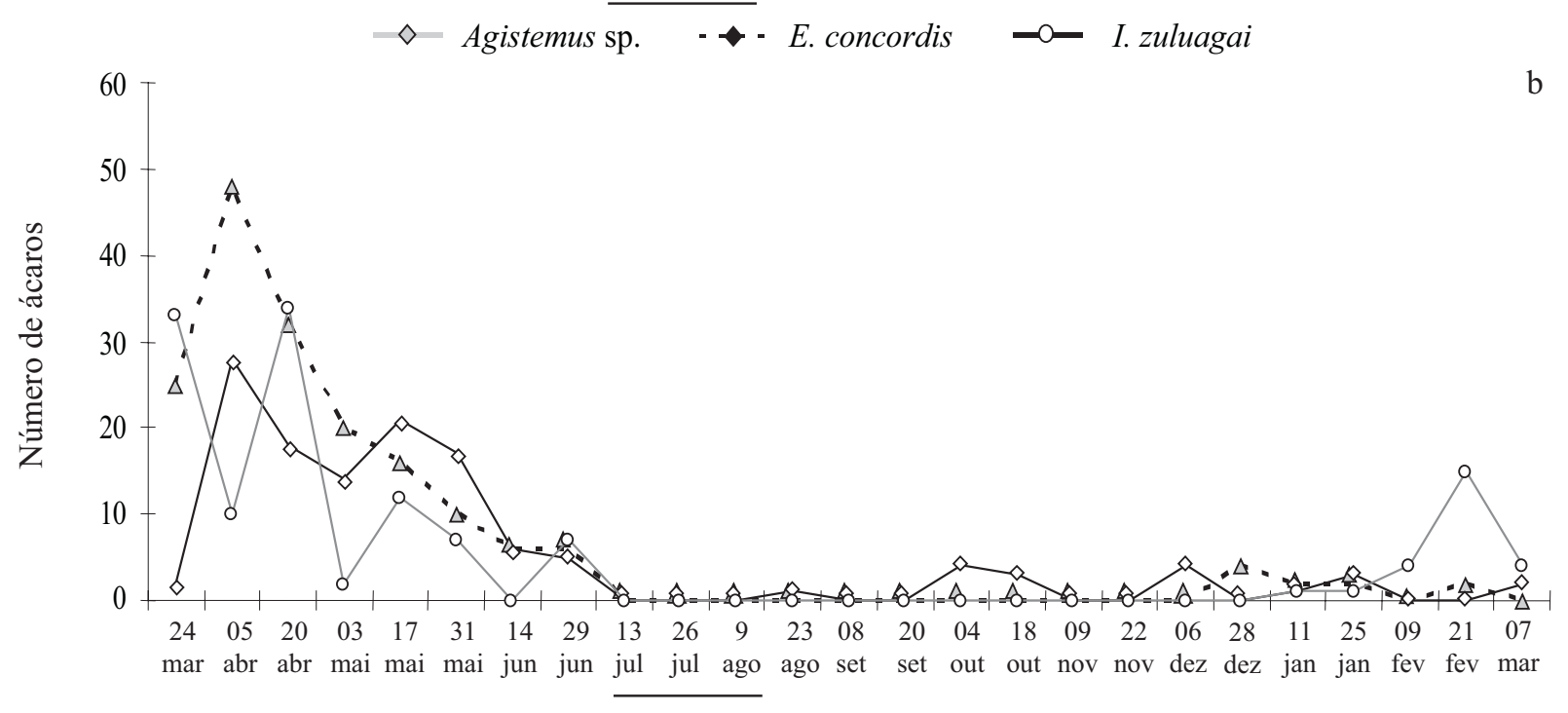

$\cdot-\Delta-$ C.duosetosus $\leadsto$ Typhlodromimus aff. amilus $\quad-0-$ Z. quasagistemas

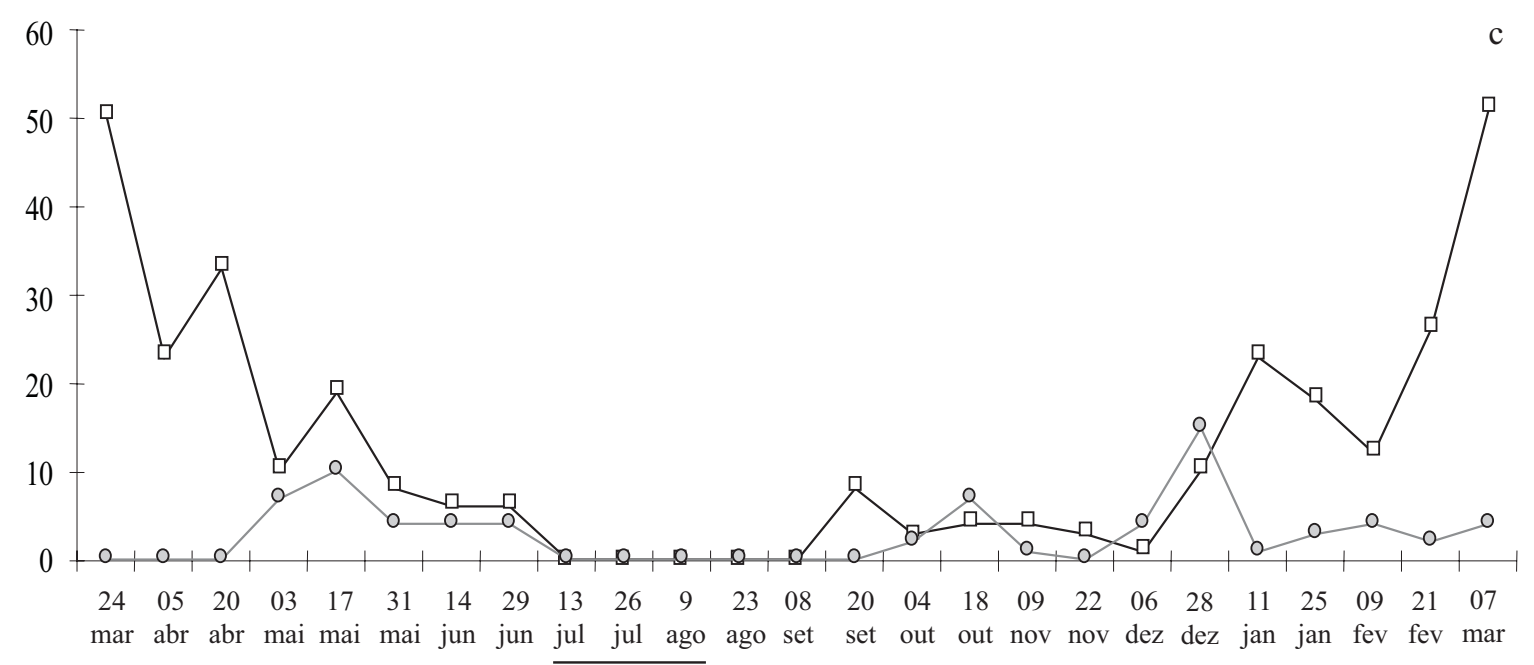

$\rightarrow \square-$ Scirula sp. $\quad=0-$ A. acalyphus

Fig. 5. Flutuação populacional dos predadores mais abundantes nos dois cultivos de seringueira de março de 2004 a março de 2005 em Itiquira, MT. Barra: período de senescência. 
manter os níveis populacionais de $T$. heveae em número reduzido. Os mesmos autores observaram $Z$. quasagistemas (citado como Z. aff. yusti) alimentando-se de T. heveae. Segundo Ferla \& Moraes (2003b), Agistemus floridanus Gonzalez (Actinedida: Stigmaeidae) apresentou alta taxa de oviposição quando alimentado com $C$. heveae e $T$. heveae. Foram observados estigmeídeos ocorrendo no mesmo microhábitat de L. formosa em folhas de seringueira, alimentandose de seus ovos (Hernandes \& Feres 2005). Isso sugere que os estigmeídeos podem também se alimentar das outras espécies de Lorryia registradas, já que também foram encontradas na base das folhas. Scirula sp. também poderia estar predando ácaros fitófagos. Trabalhos citam cunaxídeos alimentandose de ácaros das famílias Eriophyidae, Tenuipalpidae e Tetranychidae (Gerson et al. 2003).

Ácaros do gênero Amblyseius alcançaram seus maiores níveis populacionais, logo após os de $O$. gossypii e $T$. mexicanus. Ácaros deste gênero, como também dos gêneros Iphiseiodes e Typhlodromips alimentam-se de ácaros pertencentes a diversos gêneros de Tetranychidae, alguns podendo ter preferência por Eriophyidae (McMurtry \& Croft 1997). Segundo esses mesmos autores, populações de ácaros do gênero Euseius, apesar de se desenvolverem melhor na presença pólen, podem se alimentar de ácaros tetraniquídeos e eriofiídeos.

O conhecimento dos períodos em que as espécies fitófagas atingem as suas maiores abundâncias no cultivo pode auxiliar na utilização de controladores biológicos, como ácaros predadores e fungos patogênicos.

\section{Agradecimentos}

Aos engenheiros agrônomos de Plantações E. Michelin Ltda., Itiquira, MT, Cássio J. H. Scomparim e Fernando S. Fonseca, pelas informações fornecidas a respeito dos locais de coleta e ajuda durante o andamento do projeto. Ao Prof. Dr. Antonio C. Lofego (Centro Universitário do Norte Paulista - UNORP), pela identificação dos tarsonemídeos e auxílio na identificação dos fitoseídeos. Ao Dr. Aníbal R. de Oliveira (Depto. Entomologia, Fitopatologia e Zoologia Agrícola, ESALQ-USP), pela identificação dos oribatídeos. Ao Prof. Dr. Francisco Langeani Neto e ao Dr. Gustavo Quevedo Romero (Depto. Zoologia e Botânica, UNESP-São José do Rio Preto), pelas críticas e sugestões ao trabalho. A Plantações E. Michelin Ltda., pela bolsa concedida e financiamento do projeto.

\section{Referências}

Ab'Saber, A.N. 2003. Os domínios de natureza no Brasil: Potencialidades paisagísticas. São Paulo, Ateliê Editoral, 159p.

Ayres, M., M. Ayres Jr., D.L. Ayres \& A.S. dos Santos. 2003. BioEstat 3.0: Aplicações estatísticas nas áreas das Ciências Biológicas e Médicas. Belém, Sociedade Civil Mamirauá/ CNPq, 290p.

Baker, E.W. 1965. A review of the genera of the family Tydeidae
(Acarina). Adv. Acarol. 2: 95-133.

Baker, E.W. \& A.E. Wharton. 1952. An introduction to acarology. New York, MacMillan Co., 465p.

Bayan, A. 1986. Tydeidae mites associated with apples in Lebanon (Acari: Actinedida: Tydeidae). Acarologia 27: 311-316.

Bellini, M.R., G.J. de Moraes, R.J.F. Feres. 2005. Ácaros (Acari) de dois sistemas comuns de cultivo de seringueira no noroeste do Estado de São Paulo. Neotrop. Entomol. 34: 475-484

Calvert, D.J. \& C.B. Huffaker. 1974. Predator (Metaseiulus occidentalis) - prey (Pronematus spp.) interactions under sulfur and cattail pollen applications in a noncommercial vineyard. Entomophaga 19: 361-369.

Coutinho, L.M. 2002. O bioma Cerrado, p. 77-91. In A.L. Klein (org.), Eugen Warming e o cerrado brasileiro. São Paulo, Editora UNESP, Imprensa Oficial do Estado, 156p.

Demite, P.R. \& R.J.F. Feres. 2005. Influência de vegetação vizinha na distribuição de ácaros (Acari) em seringal (Hevea brasiliensis Muell. Arg., Euphorbiaceae) no município de São José do Rio Preto, São Paulo, Brasil. Neotrop. Entomol. 34: 829-836.

Fazolin, M. \& L.V. Pereira. 1989. Ocorrência de Oligonychus gossypii (Zacher 1921) (Acari: Tetranychidae) em seringueiras cultivadas. An. Soc. Entomol. Bras. 18: 199-202.

Feres, R.J.F. 1992. A new species of Calacarus Keifer (Acari, Eriophyidae, Phyllocoptinae) from Hevea brasiliensis Muell. Arg. (Euphorbiaceae) from Brazil. Int. J. Acarol. 18: 61-65.

Feres, R.J.F. 2000. Levantamento e observações naturalísticas da acarofauna (Acari, Arachnida) de seringueiras cultivadas (Hevea spp., Euphorbiaceae) no Brasil. Rev. Bras. Zool. 17: 157-173.

Feres, R.J.F. 2001. Ácaros eriofiídeos (Acari: Eriophyidae) em seringueira (Hevea brasiliensis Muell. Arg.: Euphorbiaceae) no Brasil, p. 31-36. In L.A.N. de Sá \& G.J. de Moraes (orgs.), Ácaros de importância quarentenária. Jaguariúna, Embrapa Meio Ambiente, 40p.

Feres, R.J.F., D. de C. Rossa-Feres, R.D. Daud \& R.S. Santos. 2002. Diversidade de ácaros (Acari, Arachnida) em seringueiras (Hevea brasiliensis Muell. Arg., Euphorbiaceae) na região noroeste do estado de São Paulo, Brasil. Rev. Bras. Zool. 19: 137-144.

Ferla, N.J. \& G.J. de Moraes. 2002. Ácaros (Arachnida, Acari) da seringueira (Hevea brasiliensis Muell. Arg.) no estado do Mato Grosso, Brasil. Rev. Bras. Zool. 19: 867-888.

Ferla, N.J. \& G.J. de Moraes. 2003a. Ciclo biológico de Calacarus heveae Feres, 1992 (Acari, Eriophyidae). Rev. Bras. Entomol. 47: 399-402.

Ferla, N.J. \& G.J. de Moraes. 2003b. Oviposição dos ácaros predadores Agistemus floridanus Gonzalez, Euseius concordis (Chant) e Neoseiulus anonymus (Chant \& Baker) (Acari) em resposta a diferentes tipos de alimento. Rev. Bras. Zool. 20: $153-155$.

Flechtmann, C.H.W. 1973. Lorryia formosa Cooreman, 1958 - Um ácaro do citros pouco conhecido no Brasil. Cienc. Cult. 25: 1179-1181. 
Flechtmann, C.H.W. 1986. Ácaros em produtos armazenados e na poeira domiciliar. Piracicaba, Fundação de Estudos Agrários "Luis de Queiroz", 67p.

Flechtmann, C.H.W. \& R.J. Arleu. 1984. Oligonychus coffeae (Nietner, 1861), um ácaro tetraniquídeo da seringueira (Hevea brasiliensis) novo para o Brasil e observações sobre outros ácaros desta planta. Ecossistema 9: 123-125.

Gerson, U. 1968. Five tydeid mites from Israel (Acari: Prostigmata). Isr. J. Zool. 17: 191-198.

Gerson, U., R.L. Smiley \& R. Ochoa. 2003. Mites (Acari) for pest control. Oxford, Blackwell Publishing, 539p.

Hernandes, F.A. \& R.J.F. Feres. 2005. Two new species of Zetzellia Oudemans (Acari: Stigmaeidae) that threaten the concept of genera: disgeneric marriage? Zootaxa 1048: 27-44.

Hernandes, F.A. \& R.J.F. Feres. 2006. Diversidade e sazonalidade de ácaros (Acari) em seringal (Hevea brasiliensis, Muell. Arg.) na região noroeste do estado de São Paulo, Brasil. Neotrop. Entomol. 35: 523-535.

Instituto Agronômico de Campinas. 2004. Programa Seringueira: Importância da cultura. Disponível em: $<$ http://www.iac. sp.gov.br/centros/centro_cafe/seringueira $>$. Acesso: 09 de jun. de 2006.

Jeppson, L.R., H.H. Keifer \& E.W. Baker. 1975. Mites injurious to economic plants. Los Angeles, Univ. Calif. Press, 614pp $+74 \mathrm{pl}$.

Knop, N.F. \& M.A. Hoy. 1983. Factors limiting the utility of Homeopronematus anconai (Acari: Tydeidae) in integrated pest management in San Joaquin Valley vineyards. J. Econ. Entomol. 76: 1181-1186.

Krantz, G.W. 1978. A manual of acarology. Corvallis, Oregon State University Book Stores, 509p.

Laing, J.E. \& N.F. Knop. 1983. Potential use of predaceous mites other than Phytoseiidae for biological control of orchard pests, p.28-35. In M.A. Hoy, G.L. Cunningham \& L. Knustson (eds.), Biological control of pests by mites. University of California, Special Publication, Berkeley, n. 3304, 185p.

Lindquist, E.E. 1986. The world genera of Tarsonemidae (Acari: Heterostigmata): A morphological, phylogenetic and systematic revision, with reclassification of family group taxa in Heterostigmata. Mem. Entomol. Soc. Can. 136: 1-517.

Lorenzi, H. 2000. Árvores brasileiras: Manual de identificação e cultivo de plantas arbóreas nativas do Brasil. Vol. 1, 3. ed. Nova Odessa, Editora Plantarum, 352p.

McCoy, C.W., A.G. Selhime \& R.F. Kanavel. 1967. The feeding behavior and biology of Parapronematus acaciae (Acarina: Tydeidae). Fla. Entomol. 52: 13-19.
McMurtry, J.A. \& B.A. Croft. 1997. Life-styles of phytoseiid mites and their roles in biological control. Annu. Rev. Entomol. 42: 291-321.

Pereira, A.V. \& E.B. Carvalho Pereira. 2001. Cultura da seringueira no Cerrado. Planaltina, Embrapa Cerrados, 59p.

Perrin, T.M. \& J.A. McMurtry. 1996. Other predatory arthropods, p.471-479. In E.E. Lindquist, M.W. Sabelis \& J. Bruin (eds.), World crop pests. Eriophyoid mites - their biology, natural enemies and control. Amsterdam, Elsevier Society, 643p.

Ribeiro, J.F. \& B.M.T. Walter. 1998. Fitofisionomias do bioma Cerrado, p.89-166. In S.M. Sano \& S.P. de Almeida (eds.), Cerrado: Ambiente e flora. Planaltina, Embrapa-CPAC, $556 \mathrm{p}$.

Rizzini, C.T. 1997. Tratado de fitogeografia do Brasil. 2. ed. Rio de Janeiro, Âmbito Cultural Edições Ltda., 747p.

Silveira-Neto, S., O. Nakano, D. Barbin \& N.A.V. Nova. 1976. Manual de ecologia dos insetos. Piracicaba, Editora Agronômica Ceres Ltda., 419p.

Smirnoff, W.A. 1957. An undescribed species of Lorryia (Acari: Tydeidae) causing injury to citrus trees in Marroco. J. Econ. Entomol. 50: 361-362.

Valjakka, M., E.-M. Luomala, J. Kangasjarvi \& E. Vappavuori. 1999. Expression of photosynthesis and senescence related genes during leaf development and senescence in silver birch (Betula pendula) seedlings. Physiol. Plant. 106: 302-310.

Vendramim, J.D. 1992. Pragas de viveiros e jardins clonais de seringueira e seu controle, p.65-77. In M.J.S. Medrado, M.S. Bernardes, J.D. Costa \& A.N. Martins (eds.), Formação de mudas e plantio de seringueira. Piracicaba, ESALQ, Departamento de Agricultura, 158p.

Vieira, M.R. \& E.C. Gomes. 1999. Sintomas, desfolhamento e controle de Calacarus heveae Feres, 1992 (Acari: Eriophyidae) em seringueira (Hevea brasiliensis Muell. Arg.). Cult. Agron. 8: 39-52.

Vis, R.J. de, G.J. de Moraes \& M.R. Bellini. 2006. Mites (Acari) of rubber trees (Hevea brasiliensis Muell. Arg., Euphorbiaceae) in Piracicaba, State of São Paulo, Brazil. Neotrop. Entomol. 35: $112-120$.

Wendler, R., P.O. Carvalho, J.S. Pereira \& P. Millard. 1995. Role of nitrogen remobilization from old leaves for new leaf growth of Eucalyptus globules seedlings. Tree Physiol. 15: 679-783.

Woolley, T.A. 1988. Acarology: Mites and human welfare. Fort Collins, Colorado, Library of Congress Cataloging in Publication, 484p.

Received 23/II/06. Accepted 13/VI/06. 